\title{
La seguridad alimentaria en la zona rural de El Salvador
}

\author{
Mariel Consuelo Virginia Ayala Hernández \\ Maestra en Metodología de Investigación Científica \\ Docente Investigadora, Universidad Modular Abierta, El Salvador \\ mariel.ayala@uma.edu.sv
}

Recepción: 22/03/2017

Aceptación: 02/05/2017

\section{Resumen}

La Universidad Modular Abierta presenta esta investigación con el objetivo de establecer cómo influyen los aspectos económicos, sociales y culturales en el acceso a la alimentación en las familias de la zona rural. Es así que se identificó la alimentación de las familias, el ingreso y el precio de los alimentos; se analizó la relación del nivel educativo y los hábitos alimentarios, el acceso y disponibilidad de los alimentos y se determinaron las acciones que realizan las familias para cubrir las necesidades; tomando en cuenta, cómo las personas responsables de los hogares resuelven las dificultades para cubrir dicha necesidad básica y fisiológica, garante de la sobrevivencia de las familias de la zona, que por lo general son numerosas. Se realizó un proceso metodológico que obtuvo las características de una investigación documental y de campo con enfoque cualitativo, analizando las opiniones sobre los diferentes aspectos explorados con los informantes clave del campo muestral.

Las familias de El Salvador, especialmente en la zona rural, producen el 80\% de alimentos que se consumen en el país. De ahí que la población salvadoreña padezca más hambre y tenga limitaciones en el acceso. La pobreza que agobia actualmente a los hogares de las zonas rurales de El Salvador ocasiona como consecuencia el hambre y la inseguridad alimentaria y nutricional, debido a que los ingresos percibidos por los mismos, no logran la cobertura del costo de la canasta básica alimentaria, ni suplir las necesidades básicas del ser humano (salud, educación y alimentos).

Palabras clave: seguridad alimentaria y nutricional, canasta básica alimentaria, programa mundial de alimentos

\begin{abstract}
Universidad Modular Abierta presents this research with the objective to establish how economic, social and cultural aspects have impact on the food on the rural area families. In this way, the family foods, the income and the food cots and also the educational level relationship and the food habits were identified; the food access and availability were analyzed and actions that are made by families were determined in order to fill the necessities. Taking into account how the people in charge of their homes resolve the difficulties to cover such basic and physiological necessities, to guarantee the survival of the family in the areas which are generally numerous. A methodological process that got the characteristics of a documental research, and field with qualitative approach was conducted; analyzing the opinions about the different explored aspects with the key people of the sample field.
\end{abstract}

The Salvadorian families, especially on the rural area, produce the $80 \%$ of the food that the country consumes. Hence, the Salvadoran population suffers hunger and has limitations in access. The poverty that currently burden people's homes from the rural area in El Salvador causes as consequence hunger, food and nutritional insecurity inasmuch as the incomes cannot afford the basic basket cost neither afford the human being basic necessities (health, education and food).

Key words: food security and nutrition, basic basket, World Food Programme 


\section{Introducción}

Es importante destacar que la zona rural de El Salvador se vive una realidad diferente que en la zona urbana, dado que todavía persiste el difícil acceso a la educación superior y a un empleo digno; que no todas las personas cuentan con posesión de la tierra donde habitan, y que para sembrar deben generalmente pagar arrendamiento. Es por ello que para los jefes de hogar, la alimentación de su grupo familiar se vuelve un problema, profundizándose en las familias de escasos recursos en la medida que aumenta el número de sus integrantes, en aquellas que no cuentan con un empleo e ingreso digno y las que no reciben remesas; quienes probablemente deberán hacer altos sacrificios para lograr llevar sustento a sus hogares.

Dada esta situación se indagó sobre las condiciones que enfrentan las familias en la zona rural día a día, además de analizar el desequilibrio entre el costo de los alimentos y los ingresos que reciben, acrecentando la problemática cuando en el núcleo familiar es una sola persona la que lleva los ingresos para el sustento diario.

Para obtener esta información se estableció cómo influyen los aspectos económicos, sociales y culturales en el acceso a la alimentación de las familias de la zona rural, específicamente el ingreso y el precio de los alimentos. También se analizó la relación del nivel educativo y los hábitos alimentarios de las familias con el acceso y disponibilidad de los alimentos, determinando las acciones que estas realizan para cubrir las necesidades alimentarias.

En El Salvador, al igual que en muchos países en vías de desarrollo, el acceso a la alimentación se convierte en un camino difícil para cientos de familias, pero sobre todo para quienes cumplen la función de responsables de hogares, quienes deben sortear múltiples problemas para llevar el sustento diario. Uno de los factores importantes es la pobreza que viven los hogares, principalmente de las zonas rurales, y no tienen acceso para cubrir el costo de la canasta básica alimentaria (CBA), se refiere al requerimiento mínimo calórico que necesita un individuo para desarrollar un trabajo (DIGESTYC, s.f.); mucho menos los servicios de salud y educación, dando como consecuencia serias repercusiones sobre el crecimiento y desarrollo humano. Así mismo es uno de los problemas que se vive día a día en los hogares de la población salvadoreñas en el sector de la zona rural.

La seguridad alimentaria y nutricional $(\mathrm{SAN})^{1}$ está relacionada directamente a la producción de alimentos y a las condiciones de empleos e ingreso de familias para garantizar el acceso económico a los alimentos; sin

1. Dentro del artículo, la autora se referirá a este término a través de sus siglas. 
embargo, el cambio climático y los desastres naturales plantean nuevos retos en el tema de SAN y determinan las condiciones agropecuarias del país.

Los factores económicos constituyen los principales determinantes de la inseguridad alimentaria y nutricional de la población, debido a la estrecha relación que guardan con la pobreza de El Salvador. En este sentido, la baja capacidad para generar ingresos se convierte en la principal causa del inacceso a los alimentos que posibilitan la cobertura de las necesidades nutricionales requeridas para un adecuado desarrollo humano.

En El Salvador, el grupo de la población con el mayor índice de pobreza son las personas relacionadas con la agricultura, los trabajadores agrícolas y productores de granos básicos de subsistencia. Los responsables de una buena parte de la producción de alimentos son los que más carecen de suficientes alimentos. En el caso de los productores de subsistencia, está relacionado con la falta de rentabilidad de producción de granos y su baja escala de producción.

Se considera a la soberanía alimentaria como el derecho de las naciones a alimentos nutritivos, idóneos, accesibles, originados de forma sostenible y sin detrimento del medio ambiente; además incluye el derecho a elegir su propio sistema alimentario. La soberanía alimentaria defiende los intereses de las actuales y futuras generaciones. En El Salvador, la capacidad de tierra para cultivar es limitada y los agricultores presentan severos problemas para lograr cubrir la demanda nacional y suplir sus gastos familiares.

Cabe resaltar que más del 70\% de pequeños productores del país carecen de un área suficiente para producir sus alimentos, por lo cual se ven obligados al arrendamiento de pequeñas áreas de tierra que no superan 0.7 hectáreas de extensión, en las que producen granos básicos con bajo rendimiento, al grado de que, en su mayoría, no alcanzan a cubrir sus necesidades de vender para obtener ingresos adicionales (DIGESTYC, 2005).

Los costos en los que incurren los agricultores propician un desbalance en las oportunidades de negocio para mejorar sus ingresos familiares. Es importante destacar que la soberanía alimentaria también origina procesos de comercio, que garanticen ingresos decorosos para los agricultores, y busca que los derechos de los consumidores sean respetados a fin de controlar su propia alimentación y nutrición. Garantiza los derechos de acceso a la gestión de la tierra para la producción nacional de alimentos.

A raíz de múltiples factores de índole económico y social como el desempleo, la falta de propiedad de la tierra, la poca educación, entre otros; los habitantes en la zona rural enfrentan dificultades para obtener una plena alimentación para sus familias, en donde 
a diario deben sortear muchos aprietos para llevar un poco de sustento, que en su mayoría, no implica una nutrición correcta. Por ello se hace necesario mejorar el acceso de las mujeres a los medios de producción y a la SAN, ya que son ellas las que garantizan en última instancia este elemento familiar, así como la corresponsabilidad dentro de las familias y en la sociedad.

Las causas fundamentales de la desnutrición y la inseguridad alimentaria se encuentran en la desigualdad social y de género; la pobreza, la falta de empleo, los bajos salarios e ingresos, la baja educación, las condiciones inadecuadas de producción de alimentos (tierra erosionadas por cultivos de granos básicos en laderas, alto costo de insumos y tecnología) y el alto costo de los alimentos (CONASAN, 2011). Además de la agricultura, la ganadería también se convierte en uno de los principales ingresos en la zona rural para algunos como propietarios, y otros simplemente como empleados.

\section{Materiales y Métodos}

En la investigación se requirió el diseño y aplicación de un tipo de estudio descriptivobibliográfico-documental y de campo con enfoque cualitativo, analizando las opiniones sobre los diferentes aspectos explorados en correspondencia con los objetivos y resultados esperados, desde la perspectiva de la realidad que enfrenta la población salvadoreña en la zona rural para lograr dar cobertura a la canasta básica. Para ello, se dispuso de fuentes primarias y secundarias, los cuales constituyeron importantes insumos informativos $y$ datos necesarios.

De esta manera, utilizando el diseño de investigación descriptivo, sobre la seguridad alimentaria, los diferentes componentes y dimensiones; lo cual ha permitido el análisis e interpretación de la misma, particularmente en el área rural.

Se contactó con líderes de comunidades de las zonas rurales en los municipios de San Luis Talpa y San Juan Talpa del departamento de la Paz, y el municipio de San Francisco Javier en el departamento de Usulután; quienes proporcionaron información sobre la provisión de alimentos a sus familias y lo que hacen para resolver cuando los ingresos no son suficientes; se indago con ello también, sobre aspectos socioeconómicos y culturales que intervienen para poder obtener la alimentación de los hogares en la zona rural.

Es importante destacar la indagación por vía electrónica, mediante la página web de las instituciones que velan por la seguridad alimentaria en El Salvador; además de la información y datos proporcionados de manera impresa con el propósito de conocer el aporte que realizan las instituciones que intervienen en este aspecto, mediante dichos organismos para generar estrategias que permitan mejorar la calidad de vida de los habitantes de estos sectores poblacionales. 
De la población identificada, se definió a las personas para realizar las entrevistas, en coordinación y comunicación telefónica y/o por vía correo electrónico directamente con las instituciones, a las cuales se les solicitó la delegación de personal para entrevista, considerando el criterio de los expertos que trabajan en el tema de seguridad alimentaria.

En la investigación se visitó a los informantes clave directamente a las oficinas de cada organismo e instituciones, y a las comunidades utilizando la técnica denominada entrevistas a informantes clave. Esto permitió la conversación con el personal de las instituciones sobre la seguridad alimentaria en El Salvador. De este modo, con los líderes comunales se recabó información importante para el análisis del tema. Las entrevistas se desarrollaron en forma de conversatorio sobre los factores socioeconómicos y culturales relacionados con la seguridad alimentaria.

Esta información fue respaldada con una grabación en audio, previa autorización de las personas involucradas. Para ello se utilizaron dos guías de entrevista, que contribuyeron a un mejor registro de la información y organización del trabajo investigativo. Las guías se estructuraron con preguntas abiertas y se administró a los expertos de organismos en el tema relacionado con seguridad alimentaria, y el otro para los líderes de comunidades de la zona rural.
La información fue ordenada y procesada partiendo de las respuestas escritas y audios. Se realizó la identificación de palabras clave dentro de la estructura de la respuesta de cada una de las personas, clasificándolas conforme a los objetivos planteados en la investigación. Posteriormente se procedió al análisis e interpretación de la misma, haciendo contraste con teorías, conceptos y definiciones relacionadas con el tema. La presentación de los hallazgos responde a esta lógica de estructuración mencionada.

\section{Resultados y Discusión}

La principal fuente de empleo de las personas consultadas es la agricultura, desempeñando tareas como jornaleros. Este es un empleo de tipo temporal y en él se involucra a hombres, mujeres y niños, quienes producen el $80 \%$ de los alimentos que consume la población del país; y son quienes más limitaciones presentan en su sistema alimenticio, que en muchos casos sustituyen los alimentos con la ingesta de comida chatarra.

Las familias, en alguna manera, se proveen de alimentos con las donaciones que eventualmente realizan organismos del gobierno local o nacional; las iglesias, organizaciones no gubernamentales y programas internacionales de países amigos. En cuanto al estudio de la seguridad alimentaria de la zona rural en El Salvador, los hallazgos fueron los siguientes: 
Aspectos económicos, sociales y culturales relacionados con el acceso a la alimentación en las familias

En relación al tema, la Directora Ejecutiva del Consejo Nacional de Seguridad Alimentaria y Nutricional (CONASAN) manifiesta que

la zona rural del país como un entorno social ha tenido capacidad para adaptarse a diversos escenarios sociales, en correspondencia al modelo social, político y económico que prevalece; sin embargo, esos cambios no han permitido erradicar problemas como, la falta de empleo y la pobreza. (D. de Márquez, comunicación personal, 11 de agosto de 2015).

Se menciona que, "existen en El Salvador 32 municipios en extrema pobreza severa con un promedio de $49.92 \%$ hogares en pobreza, lo que implica según el mapa de la pobreza de 175,038 habitantes" (D. de Márquez, comunicación personal, 11 de agosto de 2015). El Ing. Gerardo Merino de INCAP/OPS hace alusión a la EHOM (2013), que el 29.59\% del total de hogares en el país se considera como pobres; y compara datos del año anterior cuando refiere que, "en 2012, el dato fue de $34.5 \%$. Si se mide por zona en la rural alcanza al $35.98 \%$ de los hogares, mientras que en la zona urbana el $26.15 \%$ es registrado como pobre" (Merino, G., comunicación personal, 16 de julio de 2015)
Asimismo, una líder comunitaria manifiesta que, en la zona rural del país, la generación de empleo - además de ser limitada- no es lo que los habitantes están demandando, debiendo acudir a las ciudades en busca de mejores oportunidades lejos de la actividad agrícola, ya que los salarios en este sector son bajos e inestables, en comparación al salario en las zonas urbanas (...) cuando hay trabajo a veces se gana a diario entre $\$ 2.00$ y $\$ 5.00$ dólares, pero a veces solo se trabaja tres o cuatro días semanales, aproximadamente unos \$20 a \$25 dólares mensuales; se hacen un total de $\$ 80.00$. El problema ocurre cuando se trabaja solo dos o tres días por semana. (Ruíz, E., comunicación personal, 31 de julio de 2015).

\section{Seguridad alimentaria en el hogar: acceso a} los alimentos de las familias de zona rural

La seguridad alimentaria está relacionada directamente a la producción de alimentos a las condiciones de empleo e ingreso de las familias, y a los precios de los alimentos en el mercado para garantizar el acceso en los hogares.

En relación a la producción de alimentos, de acuerdo a la opinión del ingeniero Carlos Gómez, experto en seguridad alimentaria, haciendo referencia a la Política Nacional de Seguridad Alimentaria y Nutricional, "en el país existe una alta densidad poblacional, poca extensión territorial, alto riesgo de insuficiente producción de alimentos, y en una 
alta dependencia del exterior, especialmente en lo que se refiere a granos básicos (maíz, frijol y arroz)" (Gómez, C., comunicación personal, 14 de julio de 2015).

En este sentido,

revisando el documento de la Política, en relación a este tema se registra que la producción nacional de arroz es muy baja y se importa alrededor del 74\% del consumo nacional; en cuanto al frijol, el porcentaje de dependencia es del 39\% y de maíz blanco el 9\%. Una de las razones más importantes es que las condiciones climáticas del país, no son las mejores para la producción de este cereal.

Identifican que, dentro de los principales problemas para la producción de alimentos en la actualidad, es el monopolio que se mantiene en la comercialización de fertilizantes e insecticidas, la dependencia del exterior en suministros y tecnologías genera vulnerabilidad por su efecto sobre el empleo, los recursos y el medio ambiente.

Otros aspectos importantes son los bajos niveles de crédito destinados a la agricultura. En el 2009, solamente el 10\% de los créditos requirió una serie de requisitos más allá de las posibilidades de las familias pobres, y además los intereses fueron considerablemente altos. También una de las razones de la baja producción en el país, es la baja o nula rentabilidad de los productos, este es un aspecto que se re- laciona con la política comercial (Gómez, C., comunicación personal, 14 de julio de 2015).

En este tema, manifiesta la Viceministra de Económica, Licda. Merlín Barrera, que

los ingresos de una familia pueden estar asociados a diferentes fuentes, como remuneraciones. Especialmente en la zona rural también está relacionado a la recepción de remesas desde el exterior, además de programas de asistencia. Hay una cantidad de programas que están ejecutando por parte del gobierno, uno de ellos es el Programa de Agricultura Familiar, que atiende 10 cadenas productivas en el sector agropecuario y es una de las variantes que ha contribuido de forma significativa (Barrera, M., comunicación personal, 31 de julio de 2015).

Con la información proporcionada se evidencia la problemática que podría estar enfrentando las familias que residen en las zonas rurales de El Salvador, tal como lo menciona Barrera, haciendo relación con información de datos nacionales al decir,

dado que según datos actualizados de DIGESTYC, con los últimos incrementos la canasta básica rural podría ser aproximadamente de \$151.00 dólares. La encuesta de DIGESTYC también mide los hogares que se encuentran clasificados como pobreza extrema, que es aquella que no les permite cubrir la canasta básica. En la medición del MINEC², el 7.11\% 
de la población se encuentra bajo extrema pobreza (Barrera, M., comunicación personal, 31 de julio de 2015).

La encuesta registra que,

por otro lado, está el alto costo de la vida, que incluye el alza de los precios de los alimentos, lo cual es enfrentado por toda la población, más aún para muchas familias ni otro tipo de apoyo, donde cae el mayor peso (Barrera, M., comunicación personal, 31 de julio de 2015).

En este aspecto, según el Jefe de Visión Mundial de municipio, la opinión de los líderes comunitarios es la siguiente:

Esta situación genera graves efectos que marcan para toda la vida el bienestar social físico y mental de la niñez y adolescencia; quienes son retirados de la escuela, y consumen alimentos más baratos y menos nutritivos; refiriendo al respecto, sino se puede mandar a los hijos a la escuela no se mandan; y además, compramos alimentos de acuerdo a lo que tenemos (Fuentes, R., comunicación personal, 31 de julio de 2015).

En el área rural, obviamente la población está más expuesta, es más vulnerable; tiene que tomar estrategias más extremas para poder enfrentar la situación de inseguridad alimentaria, porque ya no logra producir suficiente o no tiene ingreso para adquirir suficiente alimentación.

\section{Relación del nivel educativo y los hábitos alimentarios de las familias con el acceso y disponibilidad de los alimentos}

Recibir educación y una alimentación adecuada son derechos humanos y ambos son determinantes en el desarrollo de una persona cuyo acceso permite una vida saludable y productiva.

En este tema se observa mucha coincidencia en las opiniones de los actores clave entrevistados, representantes de organismos relacionados con el tema de la SAN, quienes retoman datos de la EHPM del año 2013. Al decir que en El Salvador aún persiste el analfabetismo, aproximadamente 693,181 personas de diez años y más no saben leer y escribir; lo que representa una tasa de analfabetismo de aproximadamente el 14\%: en las mujeres 16\% y en los hombres el 11.6\%. Distribuido por área geográfica, se tiene que en el área urbana la tasa de analfabetismo es de 7.6\%; dentro de esta, la proporción de mujeres es de 5.2\% y la de los hombres de 2.4\%. En el área rural, la tasa de analfabetismo es de $18.9 \%$ correspondiente el $11.0 \%$ para las mujeres y el $7.9 \%$ para los hombres.

Hacen también mención de los Objetivos de Desarrollo del Milenio, suscritos por El Salvador ante las Naciones Unidas, los cuales contemplan el logro de la educación primaria universal. 
La tasa de asistencia escolar para el 2009 fue $32.4 \%$ del total de población de cuatro años y más, lo que representa un total de $1,856,171$ estudiantes que asistieron a un centro educativo formal en todo el territorio nacional (Organización de las Naciones Unidas, 2015).

\section{Medios utilizados por familias para cubrir las necesidades alimentarias}

La alimentación adecuada tanto en cantidad como en calidad es una necesidad básica y un derecho de todo ser humano. La seguridad alimentaria busca garantizar el sostenimiento alimentario de una nación; sin embargo, en El Salvador, la producción nacional de alimentos no logra ser suficiente para el abastecimiento de la demanda local, según lo manifiesta el representante de INCAP/OPS, cuando manifiesta que "en los mercados la mayoría de productos que se venden son importados de países cercanos, por tanto se encarece el precio de los alimentos" (Merino, G., comunicación personal, 16 de julio de 2015).

El creciente aumento de los precios de los alimentos y los bajos ingresos en los hogares limitan el acceso a la canasta básica alimentaria, acentuando la inadecuada alimentación y nutrición en todo el país, según declararon comunitarios de los municipios de San Luis Talpa y San Juan Talpa del departamento de la Paz; y del municipio de San Francisco Javier, departamento de Usulután. Asimismo, refieren que para solventar el difícil acceso a los alimentos, aun con las limitaciones de tierra, han ido aprendiendo a sobrevivir utilizando estrategias de sembrar en sus parcelas pequeñas y patios de sus casas, produciendo algunos granos, frutas y verduras.

De igual manera se manifiesta en los líderes comunitarios la producción en las tierras, en los granos básicos para la cosecha en frijoles, la siembra de la milpa del maíz para las tortillas, el ganado para el queso y leche; esto ayuda a la distribución necesaria en los alimentos para el consumo mismo en la comunidad. En palabras de los comunitarios: "Toda la gente trabaja para poder mantener su hogar, cultivando: maíz, frijoles, hortalizas, tomate; chile, pipián, chipilín, ejote; limones, jícama, cebollín”.

Es interesante la identificación de la estrategia de intercambio de alimentos entre vecinos de la comunidad. Esto muestra que la necesidad de sobrevivencia ha producido en la población del área rural, utilizar métodos originales de intercambio de alimentos y productos; también promueve sentido de solidaridad entre las personas.

Las familias buscan como solventar la necesidad de sobrevivencia a falta de una alimentación adecuada, acudiendo a una alimentación no nutricional como el consumo de comida chatarra. Dentro de algunos lineamientos mencionados, se debe evitar la oferta de bebidas artificiales azucaradas, golosinas y otros alimentos procesados; desalentar el consumo 
de golosinas o productos poco saludables, informando sobre los efectos dañinos en los niños por el consumo frecuente.

\section{Conclusiones}

Saber cómo se alimentan los grupos poblacionales rurales y cómo logran dar cobertura a la canasta básica en el grupo familiar; si los ingresos son limitados y si la familia es amplia es de suma importancia; ya que esto se convierte en un problema que afecta su economía familiar dependiendo del número de sus integrantes, y la realidad que enfrentan a los habitantes de las zonas rurales respecto al acceso a la alimentación.
La sobrevivencia de día a día que enfrenta la familia en la zona rural, y la falta de un empleo digno en el cual poder desarrollarse, obliga al jefe del hogar buscar otras oportunidades de empleo lejos de las actividades agrícolas.

En El Salvador persiste el alfabetismo, siendo este uno de los factores que también contribuye a la inadecuada alimentación y adquisición de correctos hábitos alimenticios. Las familias en la zona rural buscan diversas formas para garantizar el sostenimiento alimentario: el intercambio de alimentos entre pobladores, la creación de huertos caseros y escolares; y el aprovechamiento de donaciones de agencias internacionales y las remesas familiares.

\section{Referencias}

Comisión Económica para América Latina y el Caribe (2009). Estudio económico de América Latina y el Caribe 2008-2009

El Salvador, Consejo Nacional de Seguridad Alimentaria y Nutricional (2011). Política Nacional de Seguridad Alimentaria y Nutricional

El Salvador, Dirección General de Estadística y Censos (2005). IV Censo Agropecuario

El Salvador, Dirección General de Estadística y Censos (2009). Encuesta de Hogares de Propósitos Múltiples

El Salvador, Instituto de Nutrición para Centroamérica y Panamá (2006). Estudio sobre Seguridad Alimentaria en El Salvador

El Salvador, Ministerio de Agricultura y Ganadería. (2008). IV Censo agropecuario el número de productores 
El Salvador, Presidencia de la República (2011). Política Nacional de Seguridad Alimentaria y Nutricional. Recuperado de http://www.conasan.gob.sv

Estados Unidos, Consejo Estatal para el Control de los Recursos del Agua del Estado de California (2006). División Calidad del Agua, Programa de Evaluación de Monitoreo Ambiental en Agua Subterránea. Recuperado de http://www.cchealth.org/eh/smallwater/pdf/nitrate_fact_sheet_pww_es.pdf

European Food Information Council (2008). Los aditivos alimentarios. Recuperado de http:// www.eufic.org/article/es/page/BARCHIVE/expid/basic-aditivosalimentarios

Diario La Tercera (2013). Comida Chatarra: Una adicción irresistible. Recuperado de http:// diario.latercera.com/2013/03/16/01/contenido/tendencias/26-132203-9 comidadchatarra-una-adiccion-irresistible.html

Instituto Iberoamericano de Cooperación para la Agricultura (2008). La contribución del IICA al desarrollo de la agricultura y las comunidades rurales

Instituto Iberoamericano de Cooperación para la Agricultura (2015). Seguridad Alimentaria. Recuperado de http://www.iica.int/Esp/regiones/central/salvador/areas/Paginas/seguridadalimentaria.aspx

Organización Mundial de la Salud (2003). Estrategia mundial para la alimentación del lactante y del niño pequeño

Organización Panamericana de la Salud y Organización Mundial de la Salud (2009). Análisis de Situación Nacional de Salud 